\title{
Disminución de la mortalidad materna en Perú y el enfoque de capacidades
}

\section{Decreasing of Maternal Mortality in Peru and Capacities}

\author{
Carmen Avila-Jaquez (D) https://orcid.org/0000-0003-4594-8906
}

Tecnológico de Monterrey, ITESM, México, carmen_avila@hotmail.com

\begin{abstract}
This research intends to learn the capacities provided by the public policy of Peru to women, as individuals, their households or their place where they live in order to prevent maternal mortality. A brief summary of maternal health policies over the 25 years of the Millennium Development Goals is presented, underlining the most important ones such as Proyecto 2000 and ParSalud. Thirteen in-depth interviews were held with public servants, academics and activists, among others. The interviews suggested that the increase in infrastructure as well as in deliveries in which culture was respected have decreased maternal mortality.
\end{abstract}

Key words: Millennium Development Goals, maternal mortality, Peru, capacities, public policy.

Resumen: El presente estudio pretende conocer las capacidades que otorgó la política pública en Perú a las mujeres, de manera individual, a su hogar o al entorno donde habitaban, para disminuir la mortalidad materna durante los 25 años de los Objetivos de Desarrollo del Milenio (ODM). Se expone un breve resumen de las políticas de salud materna llevadas a cabo en dicho periodo, en el cual se destaca el Proyecto 2000 y ParSalud (Programa de Apoyo a la Reforma del Sector Salud). Se efectuaron 13 entrevistas a profundidad a servidores públicos, activistas y académicos, entre otros. Las entrevistas sugieren que el aumento de infraestructura y de la opción de partos donde se respetaba la cultura hizo que la mortalidad disminuyera.

Palabras clave: Objetivos del Milenio, mortalidad materna, Perú, capacidades, políticas públicas. 


\section{Introducción}

La mortalidad materna no solo es un problema médico, sino también social, económico, político, cultural, etc. El objetivo general del artículo es, primero: replantear que el aumento o disminución de la salud y el nivel socioeconómico de las personas es una relación que puede ir en ambos sentidos; pero cuando existen niveles socioeconómicos medios o bajos, la política pública puede intervenir a través del otorgamiento de capacidades para mejorar la salud. Segundo: identificar las capacidades que deben ser tomadas en cuenta por la política pública con el fin de disminuir la mortalidad materna. Tercero, analizar el caso de Perú por su destacado avance en el quinto objetivo de Desarrollo del Milenio.

El artículo está estructurado de la siguiente manera: primero se analizan algunas teorías sobre capacidades, las cuales pueden influir para que disminuya la mortalidad materna; posteriormente, se propone un marco metodológico a partir de la identificación y listado de variables e indicadores, con el propósito de facilitar el análisis y la identificación de las capacidades que se otorgaron (a las mujeres, sus hogares o entornos) en los distintos programas implementados en Perú de 1990 a 2015, destacando las políticas más emblemáticas: Proyecto 2000 y ParSalud. Debido a la falta de estadísticas a nivel desagregado, se recurre al uso de 13 entrevistas a profundidad a: funcionarios encargados del diseño e implementación de las políticas públicas (a nivel directivo), activistas o expertos en la materia, entre otros, haciendo un total de 7 mujeres y 6 hombres. Para citarlos, se menciona por primera vez su cargo para contextualizar, además de su nombre y apellido, la siguiente ocasión sólo se cita por el apellido. Asimismo, en el párrafo donde se abordan las "adecuaciones culturales" en la política de salud y se cita a varios entrevistados, las fuentes aparecen como nota al pie de página para facilitar la lectura.

\section{El otorgamiento de capacidades para disminuir la mortalidad materna}

El estudio de la salud materna y el fracaso de esta, vista como la mortalidad, es un problema con muchas aristas y también altamente evitable. Además de las cifras, su gravedad estriba en las repercusiones sociales que conlleva y en ser un indicador de la calidad de los servicios de salud en un país (Coneval, 2012: 11-22). La mortalidad materna sucede durante un periodo de la vida de las mujeres en el que son jóvenes, fértiles y productivas (aproximadamente entre los 10 y los 50 años), convirtiendo esto en una muerte prematura, donde se pierden activos humanos, los cuales dejan de contribuir durante 
su mayor potencial. Por este motivo, "mejorar la salud materna" se subió a la agenda internacional como meta por alcanzar en 25 años, de 1990 a 2015 dentro de los ODM. Los países miembros de la ONU se comprometieron, como parte de sus políticas públicas nacionales, a reducir $75 \%$ de la mortalidad materna y lograr la cobertura universal de la asistencia especializada al parto para 2015 (OMS, 2015).

Los países que más presentaban esta problemática tenían un nivel medio o bajo de desarrollo; concentrándose sobre todo en sus zonas rurales y/o pobres. En 2005, mientras que en África moría una de cada 26 mujeres, en países desarrollados fallecía una de cada 7,300 (OMS et al., 2008: 17). Para las naciones pobres, la meta era difícil de alcanzar, pues el sentido común indica que a mayores niveles económicos y de desarrollo existe una mejoría en la salud y, por lo tanto, una disminución en la mortalidad en términos generales (y de la materna en particular). Pero la evidencia empírica señala que no siempre los países ricos tienen los individuos más saludables, como sucede en Estados Unidos y Rusia (Deaton 2013: 21-22).

Sen (1999) hace este replanteamiento sobre la relación entre el desarrollo económico y la salud. Sostiene que no siempre la mayor inversión en salud da como resultado una mejor salud en las personas, porque hay quien está excluido del desarrollo, y para evitar que esto no ocurra la política pública debe intervenir (Sen, 1999: 44, 46-49). Ejemplo de lo anterior son los casos del estado indio de Kerala, de China, Sri Lanka y Costa Rica, que eran o son lugares pobres o de ingreso medio y que consiguieron incrementar los niveles de salud (Sen, 1999: 46-49). Otros ejemplos son los de Bangladesh, Camboya, China, Egipto, Etiopía, Laos, Nepal, Perú, Ruanda y Vietnam, que redujeron la mortalidad materna pese a los bajos niveles relativos de gastos en salud y en el PIB per cápita, ante los retos políticos y socioeconómicos que enfrentaron en su momento (Kuruvilla et al., 2014: 541).

Existen factores que vinculan la relación entre mayor ingreso y mejor salud, o viceversa, incluyen o excluyen a las personas de los beneficios de desarrollo y ocasionan que los índices de mortalidad disminuyan o aumenten. En 1974, Lalonde (1974: 31) identificó que los niveles de salud de una comunidad eran determinados por cuatro elementos: "biología humana, medio ambiente, estilo de vida y sistemas de asistencia sanitaria”. En 1985, Buck (1985: 10-12) hizo una crítica a Lalonde agregando más dimensiones: 1) ambientes peligrosos, 2) falta de necesidades básicas y comodidades, 3) trabajos estresantes, ingratos y despersonalizadores, 4) aislamiento y alienación y 5) pobreza. La salud está determinada por muchos factores, como "una gran variedad de procesos biológicos, sociales, económicos, culturales, tecnológicos 
e ideológicos" (Salgado y Guerra, 2014: 393-394), pero el concepto "determinantes de la salud" puede resumirse como las condiciones estructurales (que deberían ser garantizadas por el Estado o el mercado) y de vida (del individuo o del grupo) que son las principales causas de las inequidades en salud, las diferencias sanitarias, injustas y prevenibles observadas dentro y entre países (Rivera y González, 2012: 280; Salgado y Guerra, 2014: 393-394).

En la definición de Breslow (citado por Kickbusch, 2003), dichos determinantes son servicios proporcionados por el Estado, y la salud es una construcción lograda a través del otorgamiento de capacidades a las personas, es decir: los determinantes de la salud se pueden cambiar o revertir mediante el otorgamiento de capacidades (Kickbusch, 2003: 384). Estas, de acuerdo con Sen (1999), son las libertades que la gente puede disfrutar, lo que las personas valoran y tienen razones para valorar (Sen, 1999: 87). Las capacidades pueden cambiar los determinantes de la salud de manera positiva, aumentando el estado saludable de las personas.

Sen (1992 y 1999) identifica el problema de la falta de capacidades concretamente en las muertes de individuos del sexo femenino y lo llama “pérdida de mujeres". Este fenómeno es muy común en el sur de Asia, el norte de África y China, y tiene que ver con cuestiones médicas, sociales o demográficas más que con el bajo ingreso, pues la desnutrición, falta de atención en la salud y hasta la selección fetal contribuyen a acrecentar el fenómeno (Sen, 1992: 1; Sen, 1999: 20, 89, 106), aunque es bien sabido que las mujeres pobres son más vulnerables a adquirir enfermedades (Lomelí et al., 2012; Sen, 2000: 40-44).

Dheeshana y Subadra (2011) retoman las teorías de Sen (1992 y 1999) sobre el desarrollo de capacidades aplicado a la mortalidad materna. Analizan 142 países en desarrollo con la metodología de componentes principales. Su conclusión es que el desarrollo social tiene un efecto directo en la disminución de la mortalidad materna, al incrementar la capacidad reproductiva y la libertad, también a través del desarrollo de una política social (Dheeshana y Subadra, 2011:228). Asimismo, en el estudio se encontró que el desarrollo político y social tuvo impactos positivos en el desarrollo y que se deben fortalecer los esfuerzos en políticas públicas que permitan el desarrollo social, como por ejemplo: el cuidado de la salud, agua, comunicaciones, abortos seguros, etc. De igual manera, los autores concluyen que en países con bajos niveles de desarrollo se pueden reducir las muertes maternas con políticas que expandan las capacidades de la gente, en especial de las mujeres. Cabe señalar que Dheeshana y Subadra (2011) no se ciñen a capacidades específicas (pues tampoco lo hace Sen en ninguna de sus definiciones), sino 
que abren la posibilidad a todo aquello que pueda mejorar o cambiar la salud de las mujeres y eligen capacidades conforme a sus propios criterios y experiencia (Dheeshana y Subadra, 2011: 229).

Para analizar la disminución de la mortalidad materna en Perú, se propone un listado no exhaustivo sobre las capacidades que pueden influir en la salud, a manera de marco metodológico. En la teoría no se especifica qué debe ser considerado como capacidades (porque se parte del principio del otorgamiento de libertades), por lo tanto, puede haber más variables no contempladas. La lista también se basa en otras teorías, como la de los determinantes de la salud, otros "factores" y otros estudios realizados (véase el Anexo).

Se operacionalizó la variable "capacidades que deben ser otorgadas por la política pública para la disminución de la mortalidad materna”, de la cual se derivaron tres grandes dimensiones: "capacidades que deben ser otorgadas hacia las mujeres como individuos", "capacidades que deben ser otorgadas hacia los hogares donde viven las mujeres" y "capacidades que deben ser otorgadas hacia el lugar/país donde habitan".

Esta clasificación en tres grandes conceptos responde a las características propias del individuo, del núcleo familiar y a las condiciones socioeconómicas, geográficas, políticas, culturales, etcétera, que lo rodean. Posteriormente se hizo una subdivisión de las dimensiones que responde a características económicas, culturales, de salud, sociales, etcétera. De ellas se desprenden los indicadores, los cuales son las capacidades específicas que se pretenden analizar. Algunas se muestran separadas pero podrían tener relación entre sí; por ejemplo, el uso de anticonceptivos o el trabajo. Hay ocasiones en que la decisión no depende de la mujer, sino que se toma en conjunto, pareja, o de situaciones ajenas a esta (disponibilidad de métodos y costo, mercado laboral, etcétera). Se ha decidido separarlas para facilitar el análisis (véase el Anexo).

\section{La disminución de la mortalidad materna en Perú}

Perú ha sido uno de los países de América Latina que más avanzó en el quinto ODM. En 1990, tenían una Razón de Mortalidad Materna (RMM) de 251 muertes por cada 100,000 nacidos vivos, lo cual solo era superado por las naciones más pobres del continente: Haití, Bolivia y Honduras. Para 2015, Perú tuvo una RMM de 68 (Banco Mundial, 2017). Aunque la meta era de 66 defunciones por cada 100,000 nacidos vivos, fue uno de los 20 países que más habían reducido la mortalidad materna (Del Carpio, 2013: 462). 
De acuerdo con el Ministerio de Salud de Perú (2016), dentro de las principales causas de mortalidad materna en este país durante 2015 estaban: las obstétricas indirectas (33\%), hemorragia (24\%), enfermedades hipertensivas (21\%), sepsis (15\%) y las obstétricas directas (7\%). El perfil de las mujeres que morían en Perú era el siguiente: el principal lugar de fallecimiento fueron los establecimientos de salud (64\%), seguido del domicilio (25\%). La mayoría vivía en pareja: convivencia (67\%) y casadas (15.3\%). Su ocupación principal era ser amas de casa (79.9\%) y con mayor grado de estudio de secundaria (40.8\%). Sobre la edad, el 12\% tenía de 10 a 19 años (adolescentes) y el 10\%, más de 40 años. La mayoría murió durante el puerperio (63\%) (MINSA, 2016: 69).

Las cifras hablan de los resultados de la política: el Estado institucionalizó el parto y lo monitoreó a través de los controles prenatales, pues anteriormente la mortalidad se concentraba en las zonas rurales y pobres, donde no había infraestructura. La disminución de la mortalidad materna se debió, entre otras causas, al incremento del parto institucional, que en Perú, en 2012, fue de 60\% (Del Carpio, 2013: 463). Esto se atribuye a una serie de mejoras en la atención como la capacidad de resolución, la interculturalidad (implementación del parto vertical), casas de espera materna y Seguro Integral de Salud (Del Carpio, 2013: 463; Nureña, 2009: 374). Todas ellas fueron políticas de salud que el gobierno peruano puso en práctica a lo largo del tiempo.

Muchos de los programas se implementaron, se cambiaron o se quitaron, como si se tratara de una "prueba y error"; pero también esto ocurrió de acuerdo al cambio de gobierno y a las demandas de la población y sociedad civil o a las presiones internacionales. Aunque no hubo continuidad en una línea de política pública, se puede decir que los programas de salud materna iniciaron desde la época de Fujimori, cuando se le dio más impulso, ya que durante la crisis de los ochenta había disminuido la capacidad del Estado para otorgar servicios de salud (Iguiñiz y Palomino, 2012: 176). Cabe destacar que también fueron políticas que se implementaron bajo la presión de Estados Unidos para controlar el crecimiento de la población, las cuales para Perú representaban desarrollo y combate a la pobreza (Rousseau, 2007: 318-319).

En la época del presidente Alberto Fujimori (cuyo mandato duró de 1990 a 2000) se llevó a cabo el Programa Nacional de Planeación Familiar (1992-1997), que daba acceso a anticonceptivos modernos, pero terminó por promover la esterilización. Esto representó un problema de violación a los derechos de las mujeres, quienes carecían de información para tomar la decisión (Iguiñiz y Palomino, 2012: 176). Se abusó de estos programas para alcanzar las metas y acabó en esterilizaciones masivas y forzadas (Iguiñiz y Palomino, 2012: 176; Estrada y Godoy, 1996: 205; Yamin, 2003: 98). 
La política recibió muchas críticas y presiones por parte de grupos de derechos humanos. Algunos programas fueron cambiados y dejaron de ponerse en práctica; como consecuencia, el médico ex coordinador general del Programa ParSalud, Walter Vigo, relata lo siguiente:

El desprestigio de los anticonceptivos ligados a las esterilizaciones que se hicieron en el gobierno de Fujimori hizo que un grupo conservador entrara, y este grupo puso una norma que hasta ahora no se puede levantar, porque es muy fuerte: los menores de edad tienen que ir con sus padres a pedir los anticonceptivos, y eso es para públicos y privados, la norma lo exige así, si un médico es captado entregándole a una menor de edad, le pueden hacer un juicio (W. Vigo, comunicación personal, 2016).

La legislación peruana también criminaliza el aborto tanto para el paciente como para la persona que lo realiza. Con los periodos presidenciales de Alejandro Toledo (2001-2006) y Alan García (2006-2011), se puede decir que Perú entró en un periodo democrático; las organizaciones de la sociedad civil, partidos políticos y el Acuerdo Nacional consensaron para lograr planes de lucha contra la pobreza y se desarrolló el Plan Nacional Centrado en Salud (Iguiñiz y Palomino, 2012: 179; Sánchez, 2013: 30). Con García, se establecieron los "presupuestos por resultados", que, en palabras del médico-asesor del viceministro de Salud Pública y ex especialista en salud de la UNICEF Perú, Mario Tavera, daban "sustento financiero, programático y de resultado y de monitoreo anual, donde las encuestas se vuelven anuales, a los principales temas que afectan la salud de los peruanos y hubo dos prioritarios: la salud materna y neonatal y el otro la desnutrición crónica infantil”.

El presupuesto por resultados estaba "basado en la necesidad de servicios prenatales y emergencias obstétricas, como indicadores de actuación del sector salud y que sus resultados fueran auditables" (Iguiñiz y Palomino, 2012: 181); además del acceso a la información y la transparencia en el manejo del presupuesto destinado a la salud materna (Malajovich et al., 2012: 190). Cabe señalar que según la Estrategia de Cooperación Técnica OPS/OMS Perú 2010 (citada en Sánchez, 2013), Perú tiene uno de los más bajos presupuestos de Salud en Latinoamérica: 4.4\% del PIB (del cual el 2.3\% es público y el $2.1 \%$, privado), que está por debajo de la Comunidad Andina (5.8\%: Bolivia y Colombia, 8.3\%: Ecuador y 6.4\%: Venezuela) (Sánchez, 2013: 44). Más que dinero invertido, fue la rendición de cuentas y la inversión focalizada lo que logró hacer la diferencia.

Durante los años noventa, una gran cantidad de población que no estaba asegurada a los sistemas de salud formales y gratuitos para el control durante el embarazo y la atención al parto y posparto se incorporó al Seguro Público

1 M. Tavera, comunicación personal, 2016. 
de Salud (Rousseau, 2007: 316); pero aunque la incorporación de los más pobres otorgó la ampliación de derechos, saturó los servicios, como lo explica la directora del Centro de Promoción y Defensa de los Derechos Sexuales y Reproductivos, Susana Chávez:

Hay una precariedad absoluta en la prestación de servicios médicos por parte del Estado peruano, esta es suplida por el mercado y las personas tienen más gasto de bolsillo, y esto genera más inequidades en la población, y que por esta razón no se ha bajado la mortalidad materna [...] no hay que confundir el tema de la salud para todos con el aseguramiento o la carnetización de la persona (S. Chávez, comunicación personal, 2017).

Esto se debe a que "la afiliación no es prueba de cobertura" (Durán, 2012: 612), lo cual coincide con un estudio hecho en México por la Universidad de Chicago, donde los resultados concluyen que las personas afiliadas al Seguro Popular reciben una atención con menor prioridad y peor trato; y además existe una razón de mortalidad doble en estos derechohabientes, comparados con los que están afiliados a los sistemas nacionales de salud (Eng, 2014).

En Perú, el nuevo modelo de las reformas "focalizó la atención a las mujeres rurales pobres en áreas con altos niveles de mortalidad materna. En otras palabras, una mitigación sin abordar ninguna de las inequidades subyacentes" (Yamin, 2003: 33), pues las mujeres estaban muriendo por una falta de inaccesibilidad cultural o de capacidad resolutiva (Yamin, 2003: 203). Los dos programas que solucionaron el problema de la "falta de accesibilidad cultural" y "falta de infraestructura" fueron Proyecto 2000 y ParSalud, respectivamente.

Proyecto 2000 (1995-2000) fue financiado por USAID. ${ }^{2}$ Su objetivo era reducir la mortalidad materna mediante el acceso a las emergencias obstétricas, pero operó solo en algunas regiones de Perú (Seclén et al., 2003: 422; Yamin, 2003: 140). Los resultados del programa varían, pero algunos coinciden en que educó a los usuarios acerca de las señales de alarma y atención hospitalaria (Seclén et al., 2003: 433; Yamin, 2003: 268) y se establecieron estrategias de comunicación para mejorar la relación de proveedor-usuaria (Seclén et al., 2003: 433).

Para ello hubo dos estrategias: la primera se resume en palabras de la ex directora ejecutiva de Comunicaciones de Proyecto 2000, Victoria Pinedo, al decir que se fortaleció "la relación con los gobiernos locales, regionales, provinciales y a su vez convocar también a los socios estratégicos como las organizaciones sociales de enlace"; 3 y la segunda, en palabras del ex consultor

2 Las cifras son diferentes de acuerdo con distintos investigadores: $\$ 60$ millones de dólares, según Iguiñiz y Palomino (2012: 176) y \$126 millones de dólares según Yamin (2003: 121).

3 V. Pinedo, comunicación personal, 2016. 
para el Ministerio de Salud y ex director regional de Promoción y Desarrollo de Huancavelica, Raúl Choque, es la adecuación cultural microlocal y la participación comunitaria: "La participación comunitaria es un proceso a través del cual la comunidad reflexiona, analiza y decide sobre su salud, no es una imposición, pero para que haya participación comunitaria tiene que haber facilitadores que la promuevan"."

Proyecto 2000 se adecuó a la cultura en las zonas indígenas/campesinas e hizo que los gobiernos locales se apropiaran de ella y la adaptaran. La política pública otorgó la capacidad de decisión a las comunidades (el entorno donde habitan las mujeres) sobre gestionar por ellos mismos la salud de sus habitantes.

Para Nureña (2009: 374), la disminución de los índices de mortalidad materna se debió a "la adecuación cultural de los servicios, la implementación del parto vertical y el trabajo de las casas de espera". Las "casas de salud/de espera/maternas" se construyeron en áreas rurales desde 1998; en 2004 se incluyeron entre las normas y protocolos nacionales de las políticas de salud del Perú (Iguiñiz y Palomino, 2012: 180).

Las casas de espera son equipadas con "cocina y tenía un espacio también para que los chicos y los padres puedan dormir y acompañar durante el alumbramiento para que la madre se sintiera cómoda". Incluso hay espacio para que se traigan a sus animales, como lo dice el ex presidente del Centro de Promoción Familiar y Reconocimiento Natural de la Fertilidad, Martín Tantaleán: "La mujer viene con todo, las ovejitas las pone en el corral", que muchas de las veces las mujeres de la Sierra no querían ir a los establecimientos de salud, porque "las madres no tienen con quién dejar a sus hijos, o quién cuide a sus animalitos"?

No obstante, las casas de espera funcionaron en unas regiones pero en otras no, porque se usaban para las personas que presentaban complicaciones y estas eran difíciles de predecir en muchos de los casos (Yamin, 2003: 276). Tampoco funcionó por cuestiones culturales: "Siento que fracasamos en las zonas de los ríos amazónicos, el parto sigue siendo entre el 15 y el 30\% no más institucional, el resto sigue siendo domiciliario, y las mujeres se siguen muriendo". Las razones que da la médico y coordinadora nacional de

4 R. Choque, comunicación personal, 2016.

5 V. Pinedo, comunicación personal, 2016.

6 M. Tantaleán, comunicación personal, 2016.

7 V. Pinedo, comunicación personal, 2016.

8 M. Tavera, comunicación personal, 2016. 
la Estrategia Sanitaria Nacional de Salud Sexual y Reproductiva, Lucy del Carpio, son: "Porque tiene otro tipo de vivienda, otro tipo de estilo y ellos tienen inclusive muy arraigado que en algunos sitios tienen que tener el parto en el río".

Para la región andina, una de las incorporaciones sobresalientes fue el respeto a la cultura. Esto se convierte en libertad para las mujeres, es decir, en una capacidad que está otorgada por la política pública, ya que se permite llevar el parto como ellas están acostumbradas, pero vigilado por especialistas que intervienen en caso de complicaciones. La cultura se vuelve un gran determinante de la salud, sobre todo en lugares donde esta impide que las mujeres se atiendan porque únicamente el marido puede tocarlas (Santos, 2010: 25) o por malos tratos y no recibir atención en su idioma (Romero et al., 2010: 44) o "los malos tratos, la demora, los espacios muy fríos, tener a las madres mucho tiempo de piernas abiertas, decirles a las madres que son cochinas o de pronto no dejarles ingresar a su familiar al servicio de salud". ${ }^{10}$

La capacidad de otorgar un "parto intercultural" en esos lugares fue esencial para bajar la mortalidad materna. Para Nureña (2009: 372-373):

la decisión del sistema sanitario de incorporar el parto vertical no parece haberse originado en reclamos políticos de los actores sociales involucrados (de influencia apenas incipiente en el país en esos años), sino de un intento por alcanzar metas de salud pública y como forma de acción afirmativa surgida del Estado en respuesta a las críticas a los enfoques de desarrollo que dejaban de lado las perspectivas culturales. Estas críticas se veían en gran medida potenciadas por los reclamos identitarios cada vez más frecuentes en los debates políticos y académicos tanto regionales como internacionales.

Las adecuaciones culturales hicieron posible disminuir la mortalidad materna en las zonas rurales-indígenas; pero para ello se tuvieron que incorporar o cambiar muchas cosas, las cuales se detallan a continuación.

En primer lugar se incorporó el "parto vertical", 11 pues en la zona andina las mujeres "dan a luz en cuclillas" 12 y el parto es asistido por "un familiar"; 13 muchas veces es el esposo, pero la suegra es la más importante, pues "desde nuestra visión occidental el padre debe de estar presente [...] pero desde la visión andina no es la gestante la que define quién está dando el parto, sino la

9 L. del Carpio, comunicación personal, 2016.

10 V. Pinedo, comunicación personal, 2016.

11 R. Choque, comunicación personal, 2016; M. Tavera, comunicación personal 2016; L. del Carpio, comunicación personal, 2016.

12 M. Tantaleán, comunicación personal, 2016.

13 M. Tavera, comunicación personal, 2016. 
suegra". ${ }^{4}$ Se utiliza "una teja para cortar el cordón umbilical", ${ }^{15}$ ya que "en el mundo andino, se entiende que si tú cortas al niño con una navaja, el niño va a ser un delincuente" 16 en el futuro.

De igual manera se tuvieron que cambiar las salas a espacios oscuros y calientes, ya que lo frío es sinónimo de muerte; lo negro es sagrado, lo luminoso es lo contrario, como lo afirma uno de los entrevistados:

Los médicos son blancos. En la cultura andina el blanco es lo opuesto, es el terror; lo negro es lo sagrado, lo espiritual. Si tú te pones en la cabeza los campesinos y ves hacia los blancos, te das cuenta que los campesinos tienen miedo ir al centro de salud. ${ }^{17}$

Por lo tanto, si las sábanas son blancas o de color claro, "no quieren las sábanas, quieren sus mantitas y quieren en su sala de parto su pellejito"18 de borrego; es decir,

las camas para que fueran más abrigadoras, se empezaron a poner los pellejos y los cueros de las ovejas; la otra es que, de acuerdo a los periodos de la madre, se empezaron a poner frazadas tejidas (o mantas) por ellas mismas. En algunas partes de la sierra, el personal de salud empezó a hablar quechua y aimara. ${ }^{19}$

Por último, se le entrega la placenta a la familia, quien la entierra en una ceremonia, como lo explica la decana del Colegio de Obstetras del Perú, Rosa Elva Quiñones: "Hay culturas, que tú no puedes botar la placenta, se las tienes que entregar porque es parte de su cultura, lo sienten como el renacimiento, entonces la familia la tiene que enterrar", ${ }^{20}$ porque, como lo señala otro entrevistado: "Es el pago a la tierra". ${ }^{21}$

Aunque Nureña (2009) sostiene también que Proyecto 2000 tuvo un enfoque de género, y Yamin (2003) lo niega, si una política no logra empoderar a las mujeres, de nada servirá hasta que se produzca un cambio cultural:

El machismo es muy fuerte y no ha habido suficiente trabajo sobre los determinantes sociales de la salud. Los determinantes sociales deberían de haber hecho de que las mujeres de verdad que ahora van más a la primaria, van más a la secundaria, pero las mujeres no deciden sobre sus cuerpos y todavía no tiene esa capacidad, ese empoderamiento que incluye su propio cuerpo para decir estoy sangrando me voy al

14 W. Vigo, comunicación personal, 2016.

15 R. Choque, comunicación personal, 2016.

16 W. Vigo, comunicación personal, 2016.

17 R. Choque, comunicación personal, 2016.

18 L. del Carpio, comunicación personal, 2016.

19 R. Choque, comunicación personal, 2016.

20 R. Quiñones, comunicación personal, 2016.

21 M. Tantaleán, comunicación personal, 2016. 
hospital [...] eso en un análisis de repuesta de las muertes maternas, muchas de ellas llegan tarde al establecimiento porque no tenían al marido para que tomara la decisión de llevarlas o porque estaban ahí y no tenían ni un sol para movilizarse. ${ }^{22}$

Si no existe un empoderamiento de las mujeres, la política de salud seguirá siendo asistencialismo puro, pues Sen (1999: 218) y Nussbaum (2000: 2) hablan de hacer de las mujeres sus propios agentes de desarrollo. La falta de capacidad de decisión repercute en cosas complicadas como su salud y hasta en cosas sencillas como preparar los alimentos:

Por el tema de desnutrición se empoderaba a la mujer en la capacidad de decisión para preparar los alimentos porque en Perú siempre surge esta pregunta, bueno ¿qué vamos a comer hoy?, contrario a lo que uno pudiera creer [...] y su pareja generalmente buscaba alimentación que entendía que eran buenos para él y que creía que eran buenos para el niño, que finalmente eran como sopas, que no generaban nutrición, desayunar pero no necesariamente, entonces la idea era empoderar a la mujer para que ella decidiera sobre los alimentos. ${ }^{23}$

El ParSalud incidió en las regiones más pobres de Perú que tenían altos índices de mortalidad materna, es decir, "el $57 \%$ de las gestantes de las zonas rurales y el $52 \%$ de las muertes maternas del país" (Unidad de Monitoreo y Evaluación, 2012: 2). El programa estaba financiado por el Banco Mundial y el Banco Interamericano de Desarrollo, y su objetivo fue apoyar la modernización y la reforma del sistema de salud (ParSalud, 2008: 3-4). Se basó en la descentralización de los servicios, la creación y equipamiento de establecimientos con capacidad resolutoria y la distribución estratégica de estos en lugares donde eran necesarios (ParSalud, 2008: 12). Como lo explica el coordinador de la Administración Financiera del programa ParSalud, Fernando Masumura: "Se fijó el objetivo de dos horas primero porque lo podríamos costear y segundo en dos horas porque se supone que dos horas es el tiempo en el cual una mujer que tenga hemorragia puede soportar para ser atendida." 24

El programa también tenía como meta "reducir la prevalencia de anemia en gestantes de 41.5 en el año 2005 a 35 en el año 2013" (ParSalud, 2008: 41). El costo estimado del programa fue de 162,383,024 dólares, para ser ejecutado en un periodo de cinco años (Unidad de Monitoreo y Evaluación, 2012: 1).

22 R. Quiñones, comunicación personal, 20 de abril de 2016.

23 W. Vigo, comunicación personal, 2016.

24 F. Masumura, comunicación personal, 2016. 
El esfuerzo del gobierno peruano de llevar salud a las personas (capacidad individual) repercutió en la ampliación de la capacidad institucional (con la construcción de hospitales y clínicas), pero también en el otorgamiento de otras capacidades a los hogares (agua, electricidad) o al entorno (red de carreteras):

Desde que se inició la inversión en infraestructura económica y social en 1992, el sector Salud se vio favorecido mediante la construcción de postas médicas, pero sobre todo de la dotación de servicios de electricidad, agua, teléfono y carreteras, que obviamente tienen repercusiones en el mejoramiento de la posibilidad de llegar a los Cuidados Obstétricos de Emergencia en el momento oportuno (Yamin, 2003: 137).

Hay que señalar que la situación de las mujeres ha cambiado, incrementándose: el nivel de instrucción, participación en el mercado laboral y político, y el acceso al control de la natalidad y la urbanización (Yamin, 2003: 86). Cabe destacar que Perú cumplió varias de las metas de los ODM: disminución de pobreza y hambre, de la mortalidad infantil, de la tuberculosis; y aumento y atención de la planificación familiar (Kuruvilla et al., 2014: 538). Una de las respuestas de las entrevistas destaca y resume todo lo anterior:

Ha sido la mejora en la educación de la mujer, por lo menos en la educación básica. $\mathrm{El}$ analfabetismo no es que haya sido erradicado (especialmente el analfabetismo funcional), pero sí ha mejorado mucho la educación de la mujer y la participación de la mujer (es algo que seguramente en otros países del mundo ha pasado) y los procesos de urbanización en el Perú donde históricamente, pero particularmente asociado al fenómeno de la violencia senderista, que asoló el Perú entre los años ochenta y el año 94, 95, entre otras razones [...] También las razones económicas: hubo una gran migración del mundo rural al mundo urbano, y el mundo urbano permite mayor acceso a servicios, mayor información y mayor educación. El Perú también ha tenido una progresiva mejora del acceso a las vías de comunicación (eso fue ya desde la época de Fujimori), pues se empezaron a construir muchos caminos. No es todavía una situación ideal, pero en relación a como era antes, se podría decir que del 95 para atrás, la historia de lo que era para llegar a ciertos lugares en el Perú, era terrible, y después todavía hay lugares así, pero ya son los menos, y eso es clave para el tema de la maternidad. ${ }^{25}$

Algunas estadísticas nacionales peruanas que se encuentran publicadas muestran cómo estas capacidades han ido aumentando, mientras que ha disminuido la mortalidad materna. A continuación se citan algunas como ejemplos con los datos disponibles (por desgracia, en algunas no se cuenta con todos los años de los ODM): el promedio de los años de estudio alcanzados por la población femenina de 15 años y más pasó de 9.1 años en 2002 a 9.9 años en 2015 (INEI, 2018).

25 M. Tavera, comunicación personal, 2016. 
La población femenina ocupada en 2007 pasó de 6,207,000 a 7,299,000 personas en 2015, y el porcentaje de total de mujeres en edad fértil que usaban métodos anticonceptivos, que en 1991 era de 57\%, aumentó hasta alcanzar 74.6\% en 2015, impactando en una disminución de la fertilidad de las mujeres peruanas, cuya tasa pasó de 3.83 hijos en 1990 a 2.29 hijos por mujer al año final de los ODM (INEI, 2018).

En 1991, el número de hospitales era de 503, y en 2015 llegó a 651. Asimismo, el porcentaje de partos atendidos en establecimientos de salud se incrementó, al pasar de $45.5 \%$ a $91 \%$ en los años anteriormente señalados; por consecuencia, la atención prenatal por parte de profesionales de la salud, que en 1996 era de 67.3\%, alcanzó en 2015 el 97\% (INEI, 2018).

A nivel nacional, hubo un aumento en el porcentaje de los hogares que mejoraron sus condiciones de vida, como tener acceso al servicio de energía eléctrica, que en 2004 representaba el 75\%, y para 2015, el 93.8\%, o en el acceso a telefonía móvil que en 2001 era de $7.8 \%$, y para 2015 fue de $87.22 \%$ (INEI, 2018). Esto es un reflejo de la situación económica del país, pues el PIB en Perú creció. En 1994 era de 98,579 millones de soles, y para 2015 fue de 604,802 millones de soles (INEI, 2018). Con ello se puede observar que no solo fueron los programas de disminución de la mortalidad materna con enfoque intercultural y la ampliación de la capacidad institucional lo que influyó en la reducción de la mortalidad, sino también los cambios a través del tiempo de la situación de las mujeres peruanas y del país en general.

Por otra parte, no existen datos a nivel individual ni otros detalles sociodemográficos, económicos, culturales, etc., de las mujeres que murieron. En la Encuesta Demográfica y de Salud Familiar (ENDES), ${ }^{26}$ se "enfatiza la información de la salud materna infantil” (INEI, 2014: 3). La encuesta se realiza a lo largo de varios meses de seguimiento a más de 20,000 hogares y mujeres.

Con base en esa encuesta, se hacen estimaciones directas e indirectas de la razón de la mortalidad materna, utilizando el "método de sobrevivencia de las hermanas” (Maguiña y Miranda, 2013: 20). A las mujeres entrevistadas se les preguntó si tuvieron alguna hermana que murió a causa del embarazo o posterior a él y si sufría de violencia. No se preguntan más detalles. Por ejemplo, en la ENDES de 2013, de 23,5000 mujeres entrevistadas, 40 hermanas murieron durante el embarazo, 35 por aborto y 42 durante el parto, pero no en el mismo año.

26 La primera encuesta se realizó en 1986 y se volvió a aplicar por un periodo de cinco años hasta el 2000. De 2009 a la fecha se lleva a cabo cada tres años. 
Tanto Proyecto 2000 como ParSalud lograron que el personal de servicios médicos destinados a los lugares con población no hablante de español se capacitara en aprender las lenguas vernáculas; pues en Perú una parte importante de la población es indígena, alrededor del 40\% (Nureña, 2009: 369), que vive en la pobreza, analfabetismo y secuelas de la guerrilla (Gabrysch et al., 2009: 724), y por lo tanto, al ser sobre todo pobres, son altamente dependientes de los servicios de salud otorgados por el gobierno (Rousseau, 2007: 309).

Esto es importante porque las causas de muerte están ligadas a los lugares donde se vive, al nivel socioeconómico y al estilo de vida. Por ejemplo, en estudios hechos en México, se encontró que en las entidades más pobres y las zonas rurales, la principal causa de muerte son las obstétricas (hemorragia); mientras que en entidades con mayor desarrollo económico, como el norte del país y la capital, -zonas urbanas-, las principales causas de muerte son las indirectas: preclamsia y los padecimientos sistémicos subyacentes (como la diabetes) (Uribe et al., 2009: 58; Chávez et al., 2010: 68); esto da una idea general de las condiciones socioeconómicas de las mujeres, pero no dicen a detalle sobre qué capacidades les están faltando.

\section{Conclusiones}

Muchas de las estrategias y políticas llevadas a cabo en Perú para disminuir la mortalidad materna están relacionadas directa o indirectamente con otorgar capacidades. El problema es en realidad cómo medirlas. No existen suficientes datos estadísticos a nivel detalle (individual y/u hogar) para probar cuáles fueron las capacidades que la política de salud de Perú otorgó a las mujeres y qué hizo que disminuyera la mortalidad materna. Sin embargo, cabe resaltar dos hechos.

El primero es que a lo largo de los 25 años de duración de los ODM hubo un cambio a nivel nacional en las condiciones de vida de las mujeres, de sus hogares y del país en general. Hubo un incremento del nivel educativo (de la población total y en particular la femenina) y un aumento de la fuerza laboral femenina; cuando esto sucede, hay una disminución de la fertilidad que se puede observar por el aumento en el uso de anticonceptivos. Sobre las otras condiciones económicas, hubo un incremento del PIB, una mejoría en los niveles de vida de los hogares con electrificación o telefonía móvil (lo cual repercute en el aumento de las comunicaciones en caso de emergencias obstétricas, por ejemplo) y un largo etcétera, como la entrada del país a la 
democracia. Todo lo anterior creó directa o indirectamente capacidades sociales, económicas y políticas.

El segundo es que la disminución de la mortalidad materna en Perú se debe en gran medida y de manera particular a la implementación de políticas públicas de salud, que incorporaron a la población a sus sistemas sanitarios, y a la ampliación de infraestructura y mejoramiento de la capacidad institucional (creación de hospitales y clínicas, aumento y distribución del personal capacitado en lugares donde eran necesarios) y en atraer al usuario (que antes por cuestiones culturales no se acercaba a los servicios de salud), brindándole sobre todo la libertad de elegir su forma de parto, con las adecuaciones culturales. Esto también responde a una capacidad otorgada y creada por el propio gobierno en una política pública.

Las entrevistas arrojaron dos resultados importantes. El primero fue la relevancia de los programas para disminuir la mortalidad materna: ParSalud contribuyó al aumento y a la creación de la capacidad institucional por parte del Estado, y Proyecto 2000 acercó y captó población para los servicios de salud al institucionalizar el parto, mediante las adecuaciones culturales.

Esto representa una capacidad porque da la libertad a las personas para elegir. Las entrevistas arrojaron también los principales retos que ahora enfrenta Perú. Entre los muchos que hay -pero que no se mencionaron ni profundizaron en este artículo-, se encuentran: la legalización del aborto, el embarazo adolescente y el incremento de la mortalidad materna en regiones urbanas y con mayores ingresos, así como la adecuación cultural de los partos en la región de la Selva. Se debe mejorar la recolección de estadísticas de la mortalidad materna y las encuestas para tratar de incorporar la mayor cantidad de capacidades descritas en el Anexo.

Esto ayudará a las investigaciones posteriores a saber qué capacidades necesitan las mujeres antes, durante o después del parto para mejorar su salud; sobre todo, la incorporación de dichas capacidades para que sean otorgadas por la política pública en general y del empoderamiento de la mujer en particular, a fin de que sean agentes de su propio desarrollo. El Anexo, además de marco metodológico de análisis, puede servir como una propuesta para crear una política transversal que otorgue capacidades a las mujeres, sus hogares o al país e impacte en la salud materna. 
Carmen Avila-Jaquez. Disminución de la mortalidad materna en Perú y el enfoque de capacidades

\section{Referencias}

Banco Mundial (2017), Maternal mortality ratio (modeled estimate, per 100,000 live births). Disponible en: http://data.worldbank.org/indicator/SH.STA. MMRT ?order=wbapi_data_value_2010+wbapi_data_value+wbapi_data_valuefirst\&sort $=$ asc. [17 de abril de 2017].

Buck, Carol (1985), Después de Lalonde: hacia la generación de salud, Estados Unidos: Organización Panamericana de la Salud. Disponible en: http://iris.paho.org/xmlui/ bitstream/handle/123456789/32552/8598.pdf ?sequence $=1$ [ 8 de agosto de 2017].

Cartwright, Nancy y Hardie, Jeremy (2012), Evidence-based policy, a practical guide to doing it better, Estados Unidos: Oxford University Press.

Chávez, Mayra et al. (2010), "Propuesta metodológica para el estudio de la muerte materna desde la perspectiva antropológica y social”, en Perinatología y Reproducción Humana, vol. 24, núm. 1, México: Instituto Nacional de Perinatología Isidro Espinosa de los Reyes.

Coneval (2012), Evaluación Estratégica sobre Mortalidad Materna en México 2010: características sociodemográficas que obstaculizan a las mujeres embarazadas su acceso efectivo a instituciones de salud, México: Coneval.

Deaton, Angus (2013), The Great Escape: Health, Wealth And The Origins Of Inequality, Estados Unidos: Princeton University Press.

Del Carpio Ancaya, Lucy (2013), "Situación de la mortalidad materna en el Perú, 20002012”, en Revista Peruana de Medicina Experimental y Salud Pública, vol. 30, núm. 3, Perú: Instituto Nacional de Salud.

Dheeshana, Jayasundara y Subadram, Panchanadeswaran (2011), "Maternal Mortality in Developing Countries: Applicability of Amartya Sen's Theoretical Perspectives", en Journal of Comparative Social Welfare, vol. 27, núm. 3, Reino Unido: Taylor and Francis Group. https://doi.org/10.1080/17486831.2011.595071. Disponible en: https://www.tandfonline.com/doi/abs/10.1080/17486831.2011.595071 [16 de julio de 2015].

Durán, Luis (2012), "Modelo institucional de la atención a la salud en México", en Cordera, Rolando y Murayama, Ciro [comps.], Los determinantes sociales de la salud en México, México: Fondo de Cultura Económica y Universidad Nacional Autónoma de México.

Eng, Nick (2014), Making our Moms Proud: Reducing Maternal Mortality Rate in Mexico. Disponible en: http://dssg.uchicago.edu/2014/08/04/maternal-mortality-mexico. html [8 de septiembre de 2015].

Estrada, Marina y Godoy, Rosa (1996), "Género y Políticas de Salud de la Mujer en América Latina: caso Perú (Parte II)”, en Revista da Escola de Enfermagem da USP, vol. 30, núm. 2, Brasil: Journal of Sao Paulo University School of Nursing.

Gabrysch, Sabine et al. (2009), "Cultural adaptation of birthing services in rural Ayacucho, Peru”, en Bulletin of the World Health Organization, vol. 87, núm. 9, Suiza: World Health Organization. DOI: 10.2471/BLT.08.057794 Disponible en: http://www. who.int/bulletin/volumes/87/9/08-057794.pdf [16 de febrero de 2016].

Hernández, Juan y Palacio, Lina (2012), “Transición epidemiológica y el perfil de la salud”, en Cordera, Rolando y Murayama, Ciro [comps.], Los determinantes sociales de la salud en México, México: Fondo de Cultura Económica y Universidad Nacional Autónoma de México. 
Iguiñiz, Ruth y Palomino, Nancy (2012), "Data do Count! Collection and Use of Maternal Mortality Data in Peru, 1990-2005, and Improvements since 2005”, en Reproductive Health Maters, vol. 20, núm. 39, Inglaterra: Elsevier. DOI: 10.1016/S09688080(12)39605-5. Disponible en: www.rhm-elsevierDoi:10.1016/S0968-8080(12)3 9605-5 [26 de enero de 2016].

INEI (Instituto Nacional de Estadística e Informática) (2014), Perú, Encuesta Demográfica $y$ de Salud Familiar-ENDES, Nacional y Departamental 2013, Perú: INEI. Disponible en: http://www2.congreso.gob.pe/sicr/cendocbib/con4_uibd.nsf/C1C9CC0F9F25 6F1B05257D27005F7C7E/\$FILE/Encuesta_Demog_salud_familiar_ENDES.pdf [14 de marzo de 2016].

INEI (Instituto Nacional de Estadística e Informática) (2018), Estadísticas nacionales. Disponible en: https://www.inei.gob.pe/ [26 de febrero de 2017].

Kickbusch, Iona (2003), "The contribution of the World Health Organization to a New Public Health and Health Promotion”, en American Journal of Public Health, vol. 93, núm. 3, Estados Unidos: American Public Health Association. Disponible en: http:// www.ncbi.nlm.nih.gov/pmc/articles/PMC1447748/ [10 de octubre de 2015].

King, Elizabeth et al. (2008), Women and Development, Dinamarca: Copenhaguen Consensus Center. Disponible en: http://www.copenhagenconsensus.com/The\%20 10\%20challenges/Women\%20and\%20Development.aspx [11 de marzo de 2016].

Kuruvilla, Shyama et al. (2014), "Success Factors for Reducing Maternal and Child Mortality", en Bulletin of the World Health Organization, vol. 92, núm. 7, Suiza: World Health Organization. Disponible en: http://www.who.int/bulletin/volumes/92/7/14138131-ab/en/ [20 de marzo de 2016].

Lalonde, Marc (1974), A New Perspective on the Health of Canadians a Working Document, Canadá: Minister of Supply and Services Canada-Canadian Government.

Lomelí, Leonardo et al. (2012), "Niveles de vida, desigualdad y pobreza", en Cordera, Rolando y Murayama, Ciro [comps.], Los determinantes sociales de la salud en México, México: Fondo de Cultura Económica y Universidad Nacional Autónoma de México.

Maguiña, Mirtha y Miranda, Jorge (2013), La mortalidad materna en Perú, 2002-2011, Perú: MINSA, Dirección General de Epidemiología. Disponible en: http://www. unfpa.org.pe/publicaciones/publicacionesperu/MINSA-Mortalidad-Materna-Peru. pdf [20 de marzo de 2016].

Malajovich, Laura et al. (2012), "Budget transparency on maternal health spending: a case study in five Latin American countries", en Reproductive Health Maters, vol. 20, núm. 39, Inglaterra: Elsevier. Disponible en: https://www.ncbi.nlm.nih.gov/ pubmed/22789097 [26 de enero de 2016].

MINSA (Ministerio de Salud del Perú) (2009), Resolución Ministerial 27 de marzo de 2009, Documento Técnico: Plan Estratégico Nacional para la Reducción de la Mortalidad Materna y Perinatal (2009-2015), Perú: Gobierno del Perú.

MINSA (Ministerio de Salud del Perú) (2016), "Situación Epidemiológica de la Muerte Materna en Perú 2015”, en Boletín Epidemiológico, núm. 4, Perú. Disponible en: http://www.dge.gob.pe/portal/docs/vigilancia/boletines/2016/04.pdf [29 de julio de 2018].

Nureña, César (2009), "Incorporación del enfoque intercultural en el sistema de salud peruano: la atención del parto vertical”, en Revista Panamericana de Salud Públical Panamerican Journal of Public Health, vol. 26, núm. 4, Estados Unidos: Organización Panamericana de la Salud. 
Nussbaum, Martha (2000), Women and Development The Capabilities Approach, Reino Unido: Cambridge University Press.

OEA (1994), "Convención Interamericana para prevenir, sancionar y erradicar la violencia contra la mujer”, en Convención de Belem do Para. Disponible en: http://www.oas.org/ juridico/spanish/tratados/a-61.html [20 de marzo de 2016].

ONU (2015), Indicadores de los Objetivos de Desarrollo del Milenio: Portada oficial de la ONU para los indicadores de los ODM. Disponible en: https://unstats.un.org/unsd/ mdg/Data.aspx [20 de febrero de 2018].

OMS et al. (2008), Mortalidad materna en 2005, estimaciones elaboradas por la OMS, el UNICEF, el UNFPA y el Banco Mundial. Disponible en: http://www.who.int/ maternal_child_adolescent/documents/9789241596213/es/ [20 de marzo de 2016].

OMS (2015), Objetivo de Desarrollo del Milenio 5: Mejorar la salud materna. Disponible en: http://www.who.int/maternal_child_adolescent/topics/maternal/mdg/es/ [20 de marzo de 2016].

Plata, Pedro (2010), "Los vínculos entre crecimiento económico y la salud", en Perspectivas Revista de Análisis de Economía, Comercio y Negocios Internacionales, vol. 4, núm. 2, México: Universidad Autónoma de San Luis Potosí. Disponible en: publicaciones.eco. uaslp.mx/VOL6/Paper02-4(2).pdf [26 de junio de 2015].

ParSalud (2008), Resumen Ejecutivo Estudio de Factibilidad del Programa de Inversión Pública Segunda Fase del Programa de Apoyo a la Reforma del Sector Salud-ParSalud II, Perú: MINSA. Disponible en: http://www.parsalud.gob.pe/factibilidad-delprograma?...resumen-ejecutivo [16 de marzo de 2016].

Rivera, Juan y González, Teresita (2012), “Pobreza, nutrición y salud”, en Cordera, Rolando y Murayama, Ciro [comps.], Los determinantes sociales de la salud en México, México: Fondo de Cultura Económica y Universidad Nacional Autónoma de México.

Romero, Irma et al. (2010), "Muertes maternal: una revisión de la literatura sobre factores socioculturales", en Perinatología y Reproducción Humana, vol. 24, núm. 1, México: Instituto Nacional de Perinatología Isidro Espinosa de los Reyes.

Rousseau, Stéphanie (2007), "Las políticas de salud reproductiva en el Perú: reformas sociales y derechos ciudadanos”, en Revista de Estudios Feministas, vol. 15, núm. 2, Brasil: Centro de Filosofía e Ciencias Humanas e Centro de Comunicacao e Expressao da Universidade Federal de Santa Catarina. DOI: 10.1590/S0104-026X2007000200003. Disponible en: http://dx.doi.org/10.1590/S0104-026X2007000200003 [26 de enero de 2016].

Santos, Ladys et al. (2010), "Mortalidad materna: factores determinantes modificables mediante políticas públicas en la región La Libertad”, en UCV Scientia, vol. 2, núm. 1, Perú: Universidad César Vallejo.

Salgado, Nelly y Guerra, Germán (2014), "Un análisis de la investigación en México sobre los determinantes sociales de la salud: 2005-2012”, en Salud Pública de México, México: Instituto Nacional de Salud Pública.

Sánchez, Keller (2013), "Implantación de la política en salud materna en la República de Chile y Perú: Una observación desde la dimensión de gestión” (Tesis), Chile: Universidad de Chile. Disponible en: http://repositorio.uchile.cl/handle/2250/115562 [17 de marzo de 2016].

Seclén, Juan et al. (2003), "Efectos de un programa de mejoramiento de la calidad en servicios materno perinatales en el Perú: la experiencia del proyecto 2000”, en Revista Brasileira de Saúde Materno Infantil, vol. 3, núm. 4, Brasil: Instituto de Medicina Integral Profesor Fernando Figueira. 
Sen, Amartya (1992), “Missing Women: Social Inequality Outweighs Women's Survival Advantage in Asia and North Africa”, en British Medical Journal, vol. 304, núm. 6827, Inglaterra: British Medical Association.

Sen, Amartya (1999), Development as Freedom, Estados Unidos: Achor books.

Sen, Amartya (2000), Social Exclusion: Concept, Application and Scrutiny, Filipinas: Office of Environment and Social Development Asian Development Bank.

Unidad de Monitoreo y Evaluación (2012), Informe de Transferencia de Gestión Documentado ParSalud, Perú: MINSA.

Uribe, Patricia et al. (2009), "La mortalidad materna en México, estrategias y desigualdades", en Freyermuth, Graciela y Siesa, Paola [comps.], La muerte materna. Acciones y estrategias hacia una maternidad segura, México: Centro de Investigación y Estudios Superiores en Antropología Social.

Welti, Carlos (2012), “Transición demográfica y salud en México”, en Cordera, Rolando y Murayama, Ciro [comps.], Los determinantes sociales de la salud en México, México: Fondo de Cultura Económica y Universidad Nacional Autónoma de México.

Yamin, Alicia (2003), Castillos de arena en el camino hacia la modernidad. Una perspectiva desde los derechos humanos sobre el proceso de reforma del sector salud en el Perú (19902000) y sus implicaciones en la muerte materna, Perú: Centro de la Mujer Peruana Flora Tristán. 


\section{Anexo}

\section{Marco metodológico para el análisis de las capacidades de salud materna}

\begin{tabular}{|c|c|c|}
\hline Variable: & \multicolumn{2}{|c|}{$\begin{array}{l}\text { Capacidades que deben ser otorgadas por la política pública para la disminu } \\
\text { ción de la mortalidad materna }\end{array}$} \\
\hline Dimensiones & \multicolumn{2}{|c|}{ Capacidades deben ser otorgadas a las mujeres de manera individual } \\
\hline \multirow[t]{10}{*}{ Subdimensiones } & \multicolumn{2}{|c|}{ Capacidades de salud en general, de salud materna y reproductiva } \\
\hline & Indicadores & Referencia de donde surge el indicador \\
\hline & Ausencia de desnutrición & $\begin{array}{l}\text { Hernández y Palacio (2012: 123), Lomelí et } \\
\text { al. (2012: 254-255), Nussbaum (2000: 78- } \\
\text { 80), Sen (1999: 19), Plata (2010: 71-72) y } \\
\text { Romero et al. (2010: 43). }\end{array}$ \\
\hline & Vacunas e inmunización & $\begin{array}{l}\text { Dheeshana y Subadra (2011: 224) y Welti } \\
\text { (2012: 71). }\end{array}$ \\
\hline & Anticonceptivos & $\begin{array}{l}\text { Coneval (2012: 25), Plata (2010: 71-72), } \\
\text { Romero et al. (2010: 43), Dheeshana y } \\
\text { Subadra (2011: 224), Hernández y Palacio } \\
\text { (2012: 123) y King et al. (2008: 13-43). }\end{array}$ \\
\hline & Controles prenatales & $\begin{array}{l}\text { Dheeshana y Subadra }(2011: 224) \text { y Santos } \\
\text { (2010: 25). }\end{array}$ \\
\hline & $\begin{array}{l}\text { Parto humanizado y/o inter- } \\
\text { cultural }\end{array}$ & MINSA (2009: 38) y Nureña (2009: 374). \\
\hline & $\begin{array}{l}\text { Detección de enfermedades y } \\
\text { tratamientos por adicción }\end{array}$ & Hernández y Palacio (2012: 123). \\
\hline & $\begin{array}{l}\text { Captación y monitoreo de } \\
\text { embarazos en menores de } 18 \text { y } \\
\text { mayores de } 35 \text { años }\end{array}$ & $\begin{array}{l}\text { Hernández y Palacio (2012: 123), Plata } \\
\text { (2010: 71-72) y Romero et al. (2010: 43). }\end{array}$ \\
\hline & $\begin{array}{l}\text { Calidad de atención recibida } \\
\text { asociados a la salud materna } \\
\text { (ausencia de violencia obstétrica, } \\
\text { de negligencia, de tiempos de } \\
\text { espera para atención, etcétera) }\end{array}$ & Santos (2010: 25). \\
\hline
\end{tabular}




\begin{tabular}{|c|c|c|}
\hline & \multicolumn{2}{|l|}{ Capacidades económicas } \\
\hline & Indicadores & Referencia de donde surge el indicador \\
\hline & Trabajo remunerado & $\begin{array}{l}\text { Hernández y Palacio (2012: 123), } \\
\text { Nussbaum (2000: 61), Plata (2010: } \\
\text { 71-72), Sen (1992: 1), Sen (1999: 218) } \\
\text { y Welti (2012: 67). }\end{array}$ \\
\hline & Propietaria de casa o tierras & Nussbaum (2000: 78-80) y Sen (1992: 1). \\
\hline & Microcréditos/ Microfinanzas & King et al. (2008: 13-43). \\
\hline & Capacidades sociales & \\
\hline & Indicadores & Referencia de donde surge el indicador \\
\hline & Nivel educativo/años de escolaridad & $\begin{array}{l}\text { Hernández y Palacio (2012: 123), } \\
\text { King et al. (2008: 13-43), Lomelí et al. } \\
\text { (2012: 254), Nussbaum (2000: 61), } \\
\text { Sen (1992: 1), Sen (1999: 19), Sen } \\
\text { (2000: 34) y Welti (2012: 67). }\end{array}$ \\
\hline & $\begin{array}{l}\text { Ausencia de violencia (psicológica, } \\
\text { económica, sexual) }\end{array}$ & $\begin{array}{l}\text { Chávez et al. (2010: 69), MINSA } \\
\text { (2009: 34) y OEA (1994). }\end{array}$ \\
\hline Variable: & \multicolumn{2}{|c|}{$\begin{array}{l}\text { Capacidades que deben ser otorgadas por la política pública para la disminu- } \\
\text { ción de la mortalidad materna }\end{array}$} \\
\hline Dimensiones & \multicolumn{2}{|c|}{ Las capacidades que deben ser otorgadas a los hogares donde viven las mujeres } \\
\hline \multirow[t]{6}{*}{ Subdimensiones } & \multicolumn{2}{|l|}{ Capacidades económicas } \\
\hline & Indicadores & Referencia de donde surge el indicador \\
\hline & Ingreso del hogar & Santos (2010: 25). \\
\hline & Recursos destinados a la salud & Santos (2010: 25). \\
\hline & Afiliación al seguro social & $\begin{array}{l}\text { Hernández y Palacio (2012: 123), Rome- } \\
\text { ro et al. (2010: 43) y Sen (1999: 131). }\end{array}$ \\
\hline & Decisión sobre los alimentos & $\begin{array}{l}\text { Cartwright y Hardie (2012: 80-81), } \\
\text { Chávez et al. (2010: } 72 \text { y yen (2000: } \\
\text { 40-44). }\end{array}$ \\
\hline
\end{tabular}


Carmen Avila-Jaquez. Disminución de la mortalidad materna en Perú y el enfoque de capacidades

Capacidades de infraestructura

\begin{tabular}{ll}
\hline Indicadores & Referencia de donde surge el indicador \\
\hline Agua y drenaje & $\begin{array}{l}\text { Hernández y Palacio (2012: 123), Lomelí et al. } \\
(2012: 252-255) \text { y Welti (2012: 67). }\end{array}$ \\
\hline Electricidad & Dheeshana y Subadra (2011: 224). \\
\hline Teléfono e Internet & Dheeshana y Subadra (2011: 224). \\
\hline
\end{tabular}

Capacidades culturales

Indicadores

Referencia de donde surge el indicador

Ausencia de impedimento o Santos (2010:25).

restricción para que la mujer

sea atendida

\begin{tabular}{|c|c|c|}
\hline Variable: & \multicolumn{2}{|c|}{$\begin{array}{l}\text { Capacidades que deben ser otorgadas por la política pública para la disminu- } \\
\text { ción de la mortalidad materna }\end{array}$} \\
\hline Dimensiones & \multicolumn{2}{|c|}{ Las capacidades que deben ser otorgadas al lugar/país donde habitan las mujeres } \\
\hline \multirow[t]{7}{*}{ Subdimensiones } & \multicolumn{2}{|l|}{ Capacidades económicas } \\
\hline & Indicadores & Referencia de donde surge el indicador \\
\hline & $\begin{array}{l}\text { Condiciones laborales adecua- } \\
\text { das para embarazo y lactancia }\end{array}$ & Hernández y Palacio (2012: 123). \\
\hline & $\begin{array}{l}\text { PIB, porcentaje de gasto en } \\
\text { salud y seguridad social }\end{array}$ & $\begin{array}{l}\text { Dheeshana y Subadra }(2011: 224) \text { y Sen } \\
(1999: 10,39) \text {. }\end{array}$ \\
\hline & \multicolumn{2}{|l|}{ Capacidades sociales } \\
\hline & Indicadores & Referencia de donde surge el indicador \\
\hline & $\begin{array}{l}\text { Ausencia de discriminación por } \\
\text { origen racial o étnico }\end{array}$ & Welti (2012: 67). \\
\hline
\end{tabular}


Capacidades de infraestructura

\begin{tabular}{|c|c|}
\hline Indicadores & Referencia de donde surge el indicador \\
\hline $\begin{array}{l}\text { Acceso menor a dos horas de } \\
\text { distancia a hospitales y centros } \\
\text { de salud sin importar tamaño } \\
\text { de localidad (urbano o rural) }\end{array}$ & $\begin{array}{l}\text { Hernández y Palacio (2012: 123), Lomelí et } \\
\text { al. (2012: 252-255) y Welti (2012: 67). }\end{array}$ \\
\hline $\begin{array}{l}\text { Atención del parto por } \\
\text { personal calificado (médicos, } \\
\text { obstetrices, parteras) }\end{array}$ & $\begin{array}{l}\text { Santos (2010: 25) Dheeshana y Subadra } \\
\text { (2011: 224). }\end{array}$ \\
\hline $\begin{array}{l}\text { Tecnología disponible para } \\
\text { tratar complicaciones en el } \\
\text { embarazo, parto o puerperio }\end{array}$ & Lomelí et al. (2012: 254). \\
\hline $\begin{array}{l}\text { Sistemas de referencia y } \\
\text { coordinación entre los niveles } \\
\text { de atención hospitalaria, } \\
\text { disponibilidad de información, } \\
\text { expediente clínico en red }\end{array}$ & $\begin{array}{l}\text { Coneval (2012: 18), Seclén-Palacín et al. } \\
\text { (2003: 433). }\end{array}$ \\
\hline \multicolumn{2}{|l|}{ Capacidades políticas } \\
\hline Indicadores & Referencia de donde surge el indicador \\
\hline $\begin{array}{l}\text { Democracia, gobernanza y } \\
\text { estado de derecho, libertad y } \\
\text { participación política }\end{array}$ & $\begin{array}{l}\text { Deaton (2013: 14), King et al. (2008. 13- } \\
\text { 43), Nussbaum (2000: 61), Sen (1995: 10) y } \\
\text { Welti (2012: } 74) \text {. }\end{array}$ \\
\hline Acceso al aborto legal & Dheeshana y Subadra (2011: 224). \\
\hline
\end{tabular}

Fuente: Elaboración propia a partir de las referencias descritas en el mismo cuadro.

Carmen Avila-Jaquez. Doctora en Política Pública por el ITESM, México. Maestra en Administración Pública y Políticas Públicas por la misma institución. Líneas de investigación: el empodermiento de mujeres indígenas y los programas de combate a la pobreza, y el enfoque de capacidades para mejorar la salud materna. Publicaciones recientes: Avila-Jaquez, Carmen (2009), "Empowerment of women through Political Participation: Oportunidades' performance in an indigenous community of Oaxaca”, en Perspectivas Sociales/Social Perspectives Journal, vol. 11, núm. 1-2, México: Universidad Autónoma de Nuevo León; y Avila-Jaquez, Carmen y Gabarrot, Mariana (2009), "Retos locales de la política social en México: el funcionamiento del Programa de Desarrollo Humano 'Oportunidades' en una comunidad indígena bajo el sistema de gobierno de usos y costumbres”, en Confines, vol. 5, núm. 9, México: Instituto Tecnológico y de Estudios Superiores de Monterrey. 